\title{
How to Strengthen the Social Security Management Capacity Building at the Grassroots Level
}

\author{
Xiaobo Sun \\ College of Politics and Public Administration, Hainan University Hainan, Haikou 570100
}

Keywords: grassroots government; social management; capacity building

\begin{abstract}
At present, China's grassroots government has many problems in social security management, such as simplification of management body, incoordination of environmental safety construction, wide gap between urban and rural social security and the existence of formalism. Therefore, it is necessary to strengthen the grassroots government's innovation in social security management capabilities, establish a diversified social security management mechanism, increase investment in social security management, optimize the social security management structure, and improve safety emergency management capabilities of person in various departments so as to provide guarantee for the construction of the grassroots government's social security management capacity.
\end{abstract}

\section{Introduction}

The grassroots government is the key to social security management, they have the greatest number of contacts with the people and social and public utilities, and they face the most difficult situation in social security management. On the one hand, they have limited public resources for social security management. On the other hand, the complexity and numerous emergencies in basic social security management services pose challenges to the grassroots government's social security management system, service awareness, and emergency level. Therefore, it is of great significance to explore how to strengthen the social security management capacity building at the grassroots level in the new era.

\section{Problems in social security management of basic-level government}

\subsection{Simplification of management body}

In the social security management, basic-level governments play the leading role whose autonomy is severely administrative. The single management body, which is dominated by the grassroots government, leads to a sudden increase in its administrative pressure, and failure in fully mobilizing and utilizing limited manpower and financial resources. However, the public and social organizations cannot participate in social security management, leading to management gaps in some areas. The absence of social public organizations at the grassroots level reduces the efficiency and quality of basic-level government's social security management.

\subsection{Uncoordinated environmental construction}

The construction of good public environment is the foundation for implementing social security management. The awareness of social security management centered on economic construction is weak. basic-level government focuses on projects, business talks and investment promotion, but neglects the importance of regional soft environment construction. The lack of government social security management and public service functions and insufficient attention to people's livelihood have led to public disorder and threats to public security. The sustainable economic development requires the basic-level government to accurately locate its own functions and timely return to social service functions. 


\subsection{The large gap between urban and rural social security}

Social security of urban residents shall be generally institutionalized, standardized, and welfare treatment is favorable, and the safeguard items shall be complete basically. Social security of rural residents faces the dilemma of low project, low level and poor guarantee, resulting in an awkward situation. The dual structure of urban and rural areas is a long-term problem for the social security management of China's grassroots government. Although China highly promotes the integration of urban and rural areas and reduces the social security gap between urban and rural residents, the limitation of the public resources available at the grassroots level, which cannot solve the urban and rural dual problems in a short term.

\subsection{Formalism is not eradicated}

Grassroots government social security management fundamental purpose is to serve the masses, maintain the public interest, but some grassroots government to borrow the name of social performance engineering, government performance and results over the social and public interests, formalism mistaken ideas are still not eradicated. Some grassroots government officials get rid of the actual demand of local society, which leads to the decrease of the public trust in the government, influencing the image of the government, and the public relations. The value of social welfare also conflicts with the personal performance promotion of officials. The primary government should strengthen the service consciousness, locate the public function position accurately, and eliminate formalism fundamentally.

\section{Causes of problems in social management of grassroots governments}

\subsection{Ideological factors}

Ideology is the fundamental idea that governs the social management activities of the grassroots government, therefore, ideological deviation is the essential cause of various social security management problems. The false consciousness of the supremacy of government, the lack of service awareness and the indifference of legal concept let the relevant legal supervision of social security management activities from grassroots government can not be used effectively. Even the laws and regulations and legal system used to restrict grassroots civil servants have become a decoration, which cannot restrain the behaviors of grassroots civil servants.

\subsection{Social objective factors}

The problems caused by the transformation and integration of social structure and social form in the process of China's economic development pose challenges to the security management of the grassroots government. The grassroots government should bear the social security management index and pressure imposed by the superior, and face the new social problems independently; Limited public resources such as personnel, financial resources, technology and material resources owned by the grassroots government, the lacks of safety management concept and safety management experience in grassroots government leads to social security management gap in some areas; what's more, the honest mistakes of the grassroots government aggravates the contradiction between the society and the government.

\subsection{Financial burden factors}

The basic-level government's social security management funds mainly come from the central financial support, while the administrative cost in our country remains a high level, but the funds allocated to the grassroots government under huge financial burdens are very limited. This affects the optimization of social security management and the construction of soft environment. At present, the debt ratio of the township and township governments in our country continues rise up gradually, and the shortage of public resources has affected the actual social security management effectiveness of grassroots governments. 


\subsection{Management System Factors}

The reform of the government's management system in China has a heavy slogan but underestimate practice, focusing on the aim underestimate process, and the drawbacks of focusing on forms and performances have made it difficult to advance system reforms. The failure of the government management system reform to determine the relevant social security management system has not yet formed. However, the absence of supervisory bodies for grassroots civil servants' behavioral activities makes it impossible to guarantee the effectiveness of social management. At the same time, the paradox of traditional social security management models has caused the grassroots government to lose the execution power of institutional formulation and social behavior supervision.

\subsection{Management Method Factor}

The grassroots government is the main target of social security management. People's ideology, values, and cultural level are changing in the new era. The traditional planned economic system management method does not meet the requirement of social development. The grassroots government should accurately position the service role and establish public servants image and strengthen the awareness of the masses. The lack of innovation in social security management methods affects the efficiency of social security management, restricts social reform and progress, and is not conducive to the participation of the people and social organizations in political affairs.

\section{The method to strengthen the social security management capacity building of the grassroots government}

\subsection{The Establishment of multi-participation mechanism for social management}

The pattern of a single social security management subject of the grassroots government should be broken, public participation shall be mobilized, and the initiative of social organizations and the masses should be given full play. People's direct participation in the political system should be improved, people's voting rights, letters and visits, participation in the political power should be ensured and the direct communication channels between the grassroots government and the people should be broaden and maintained. To develop ways for people to participate in politics indirectly, encourage the participation of social self-organizing organizations and public welfare organizations, and give full play to the social management power of non-governmental organizations.

\subsection{Increase the financial investment of grassroots governments}

Allocate the ratio of financial input from the central government and governments at all levels and define the scope of power and responsibility of grassroots governments rationally, specify the financial investment for social security management services, and construct a fiscal distribution system in which the authority and financial power are unified. Optimize the fiscal management structure of the government and reduce the level of financial management. Optimize the allocation of fiscal funds to social welfare and infrastructure construction; Increase financial support for medical insurance, education, public health and other fields and increase the efficiency of the use of fiscal funds. Attracts social organizations and social enterprises to invest in social and public utilities through policy preferences to meet the needs of social security management and construction.

\subsection{Optimize the basic social security management structure and staff organizations}

Rationally optimize the integration of grassroots government organizations, reduce fiscal expenditures, and accumulate funds; Improve transparency of social security management and strengthen supervision. Streamline and consolidate departmental organizations according to the current status of social security management, improve the ability and efficiency of collaboration and collaboration between departments and agencies, clarify the scope of power and responsibility of various agencies, improve the organization of social security management, and establish a 
comprehensive grassroots government social management network system. Strengthen grassroots government's institutional security status. In addition, optimize human resources management and increase the professional quality of grassroots civil servants.

\subsection{Improve emergency management capabilities of grassroots government}

Except for the systematic social management capabilities, grassroots governments should also improve their emergency management capabilities. Emergency management agencies and emergency management teams were established to strengthen the core position of unified operations command. Combining grassroots government emergency response platforms with national comprehensive emergency response platforms to exert linkage effects. Establish an emergency warning detection mechanism, increase the detection of different types of public risk warning, accurately identify the authenticity of information, and ensure the effectiveness of emergency information. In the meantime, construct an emergency resource reserve system, which reserve social assistance and social service resources for emergencies.

\subsection{Innovative social security management methods of grassroots government}

Starting with concepts, methods, technologies and so on, change social management ideas, abandon the idea of authoritarianism, listen to more public opinions, strengthen the image of government authority, and broaden social security management ideas of government. Strengthen the leadership position of the grassroots government, improve the construction of laws, regulations and social systems, and guide the public to return to the correct socialist values.

\section{Conclusion}

The grassroots government is a direct participant in social security management. How to improve social security management capabilities is a problem that needs to be solved by the grassroots government. Only by abandoning the idea of unhealthy governance, emancipating active management ideas, and innovating management techniques can we strengthen the building of grassroots government social security management capabilities.

\section{References}

[1] Ma Wenfeng. Practical Research on Application of Positive Psychology in College Physical Education[J]. Wisdom. 2014; 30: 101.

[2] Tan Xiangqun. Construction of Local Practical Teaching Resources System of Applicationoriented Ideological and Political Education Courses in Colleges and Universities_-A Case Study of South Anhui Province[J]. Journal of Huangshan University. 2015, v.17; No.16002:97-101. 